\title{
COMMUNICATIONS
}

\section{SITE OF OBSTRUCTION TO AQUEOUS OUTFLOW IN CHRONIC SIMPLE GLAUCOMA*}

\author{
BY \\ J. S. SPEAKMAN $\dagger$ AND T. S. LEESON $\ddagger$ \\ University of Toronto
}

THE site of obstruction to aqueous outflow in chronic simple glaucoma must lie between the anterior chamber and episcleral plexus, yet opinion remains divided between those who believe that the obstruction occurs while aqueous passes through the trabecular meshwork before entering Schlemm's canal and those who believe that it occurs in the scleral plexus beyond the canal. Support is given to the latter view by the vascular changes associated with diurnal variations in intra-ocular pressure (Duke-Elder, 1955), by the infrequent appearance of blood in Schlemm's canal on gonioscopy in glaucomatous eyes, and by the direct measurement of pressures within the canal of Schlemm in the eyes of normal monkeys (Perkins, 1955). The altered blood flow in laminated aqueous veins following compression of episcleral vessels in glaucomatous eyes (Goldmann, 1949), and the direct measurement of resistance to outflow in normal eyes after removal of the trabecular meshwork (Grant, 1958), support the former view. Morphological studies which have attempted to establish the site of obstruction have been handicapped by the difficulty in obtaining rapidly-fixed glaucomatous material at an early stage in the disease, and by the variations in normal structure which have made observations of pathological change difficult to assess. Various authors have reported abnormalities within the trabecular meshwork, the canal, or the scleral plexus. In the meshwork, obstruction has been attributed to spongy or foamy degeneration of the cytoplasm (Flocks, 1959; Becker, 1959), to granular degeneration of collagen, and to proliferation of endothelial cells (Teng, Paton, and Katzin, 1955). Trabecular sclerosis has been described (Theobald and Kirk, 1956; Kornzweig, Feldstein, and Schneider, 1958), although it is not clear which portion of the fibres becomes sclerotic, nor has a distinction been made between thickening due to swelling and that due to fibre proliferation. A uniform thickening of the clear zone has been described in trephine biopsies among other changes in the meshwork (Unger and Rohen, 1960), and proliferation of the so-called curly collagen in the clear zone has been found in one case of glaucoma (Garron, 1959a). Adhesions

\footnotetext{
- Received for publication November 23, 1961. † Department of Ophthalmology. ‡ Department of Anatomy. 22 
between the walls of Schlemm's canal, narrowing and obstruction of the / vessels forming the scleral plexus (Theobald and Kirk, 1956), and alterations in the appearance of the trabecular nerves (Wolter, 1960) have also been described. Thus there is not only a difference of opinion regarding the major site of obstruction but morphologists cannot decide whether the primary pathological changes in chronic glaucoma are cellular or extra-cellular. In fact, it has been suggested that chronic glaucoma in its early stages may be a functional disorder with no visible abnormality (Ashton, 1960).

Detailed knowledge of the normal structure of the trabecular meshwork .has increased greatly as the result of electron microscopic studies (Garron, 1959b; Holmberg, 1959; Leeson and Speakman, 1961) and of the examination of teased wet preparations (Speakman, 1959, 1960). These studies have defined more precisely the extra-cellular components of the meshwork and the shape and size of the ultimate drainage channels and openings in the endothelial meshwork which forms the trabecular wall of Schlemm's canal. A nodular dystrophy involving the innermost trabecular lamellae has been recently described in normotensive eyes, which consisted of fusiform, nodular, and annular expansions of the clear zone (Speakman, 1961, 1962). These dystrophic nodular changes were thought to be similar in nature to the HassallHenle bodies found at Schwalbe's line in older individuals, and it was suggested that an extension of the proliferative changes throughout the meshwork might lead to increased intra-ocular pressure. It has since been possible to examine by light and electron microscopy two eyes from patients with chronic simple glaucoma (Leeson and Speakman, 1962). The findings, which are reported in detail in this paper, support the hypothesis that obstruction to aqueous outflow in chronic glaucoma is the result of proliferation and degeneration of the extra-cellular fibres in the clear zone which leads to a progressive reduction in the calibre of the drainage channels throughout the trabecular meshwork.

\section{Clinical Histories}

The following case histories are given in detail to establish the basis for the diagnosis of chronic simple glaucoma.

Case 1, a white female aged 83, was first seen at the eye clinic in 1946 and has been under observation by one of us (J. S. S.) since 1955 . The patient has never experienced haloes around lights or had an acute attack of congestive glaucoma. At the time of her first visit, glaucoma was suspected because of the appearance of the discs, but intra-ocular pressure readings were equivocal and the peripheral fields were full in each eye.

Right Eye.-In 1951 she was again examined because of blurred vision and an intraocular pressure of $35 \mathrm{~mm}$. $\mathrm{Hg}$ was found in the right eye, associated with marked cupping and field loss. In spite of miotic therapy field loss progressed, and the intra-ocular pressure remained elevated in the right eye, so that a cyclodialysis was performed in May, 1953. After a year the tension became normal in the right eye but central fixation was lost and vision was reduced to hand movements and light perception in the temporal field. 
In 1958 the right eye became painful and photophobia and tearing developed. This appeared to be due to a localized area of bullous keratitis which involved about one quarter of the cornea in the region of the cyclodialysis where a loose strip of Descemet's membrane lay curled in the anterior chamber. At the time of enucleation the intra-ocular pressure in the right eye was $23 \mathrm{~mm}$. $\mathrm{Hg}$ (Schiötz, 1955 scale) and vision was reduced to light perception only in the temporal field. The anterior chamber was clear and gonioscopy showed an open angle with an average amount of pigmentation. The iris appeared healthy but the pupil would not dilate because of posterior synechiae which developed following the cyclodialysis. The lens showed cortical opacities and the fundus was not visualized.

Left Eye.-In spite of miotic therapy, this eye showed progressive field changes (Fig. 1) and in 1959 a filtering procedure was performed. This was followed by an intra-capsular cataract extraction, complicated by vitreous loss when the sutures were removed and an updrawn pupil. An optical iridectomy improved the vision.

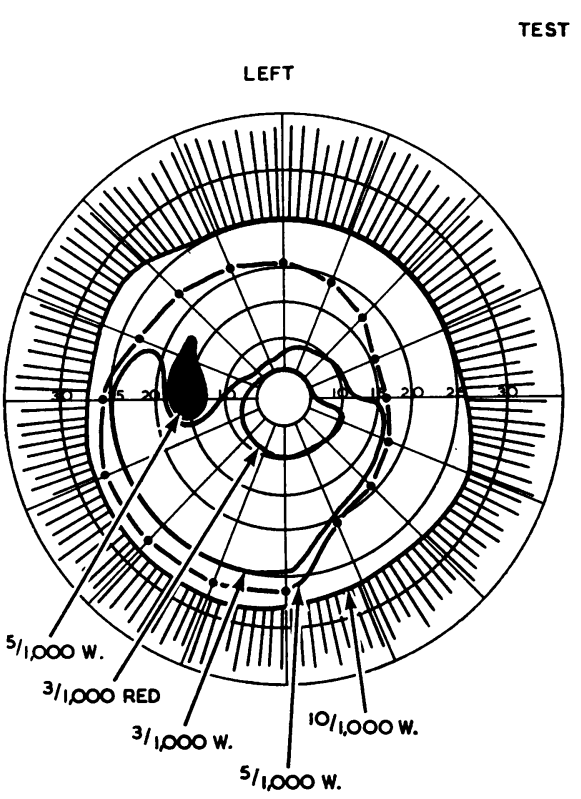

Visual Acuity: 20/70 with connections.

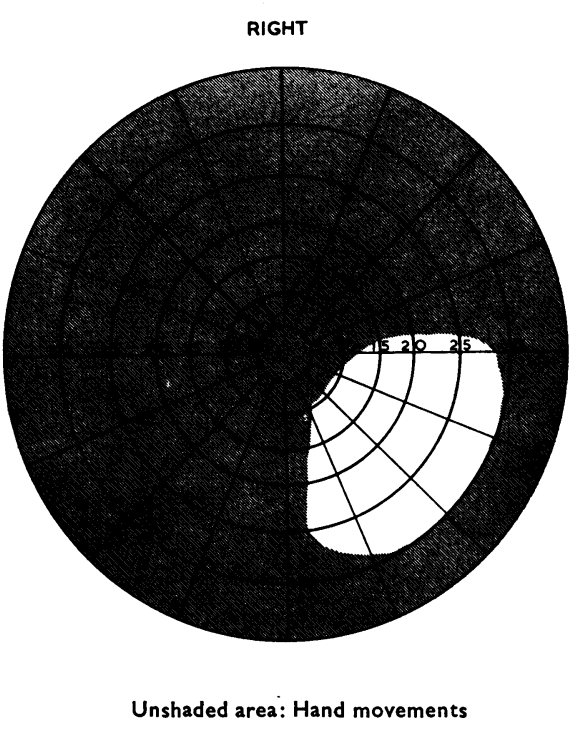

Visual Acuity: Hand movements at 1 foot

Fig. 1.-Visual field of Case 1, 8 years after beginning treatment for chronic glaucoma.

Since the enucleation of the right eye the patient has been more comfortable, but she is developing a localized bullous keratitis at the site of the cataract section in the left eye.

Case 2, a 68-year-old white female, was found to have glaucoma when she complained of blurred vision in 1956 and had used miotics intermittently since then. She was first examined by one of us (J. S. S.) in February, 1961, when she stated that she continued to have episodes of blurred vision, head pain, and haloes around lights even on pilocarpine therapy, but there was no history of an acute attack. The visual acuity was $20 / 30$ in the right eye and no perception of light in the left, and she thought that the left eye had been 
blind for 2 or 3 years. The anterior chambers were clear and gonioscopy showed narrow open angles, more pigmented in the left eye than in the right. The lenses were clear and ophthalmoscopy showed bilateral glaucomatous cupping but no retinopathy.

Right Eye.-While the patient was in hospital for assessment, the intra-ocular pressure fluctuated in the right eye from 20 to $45 \mathrm{~mm}$. $\mathrm{Hg}$, settling to around 25 on phospholine iodide 0.25 per cent. twice daily. The visual field, with a $3 / 1000$ white test object, was reduced to a semicircle below fixation extending to the $10^{\circ}$ meridian. Gonioscopy showed open narrow angles when the tension was raised.

Left Eye.-The ocular tension fluctuated from 30 to $60 \mathrm{~mm}$. $\mathrm{Hg}$, with no evidence of congestion even when the pressure was high. The eye was removed to avoid the development of painful secondary changes and the patient has since been more comfortable.

\section{Methods}

Immediately before enucleation the anterior chambers of each eye were decompressed by an ab externo incision at the limbus in order to produce a reflux of blood into Schlemm's canal and the outer layers of the meshwork. The first eye was fixed by injecting chilled buffered osmic acid (Palade) into the anterior chamber, 30 seconds after cutting the optic nerve. The anterior segment was removed and divided into two, half was left in the osmic acid and half was immersed in 10 per cent. formalin together with the posterior segment. The anterior segment of the second eye was divided into equal thirds immediately after removal, and fixation was completed by immersion in chilled buffered osmic acid, potassium permanganate 2 per cent. and 10 per cent. formalin. After 3 hours of fixation, blocks of trabecular tissue to be used for electron microscopy were removed under a dissecting microscope and techniques previously described were employed for sectioning and imbedding (Leeson and Speakman, 1961). Wet preparations of the uveal, corneo-scleral, and endothelial meshwork were prepared by techniques previously described (Speakman, 1960), and paraffin sections of the anterior and posterior segments were made, using standard procedures.

\section{Gross Examination}

The anterior segments of both eyes were examined under a dissecting microscope and an open angle was found in each, with no evidence of peripheral synechiae (Fig. 2a, $b$, opposite). The pigmentation of the filtration angle was more marked in the second eye. The posterior segment of the first eye was examined immediately after fixation revealing a pale cupped disc, normal vasculature, and no retinopathy. The osmic acid fixative turned the specimen black preventing further gross study. The second eye also showed a pale cupped disc and in addition several flecks of hard exudate and a few small fresh haemorrhages which were attributed to the trauma of rapid decompression and enucleation. The trabecular tissues in the first eye showed the usual elasticity and held together during dissection, but in the second eye the lamellae were very friable. 
(a)

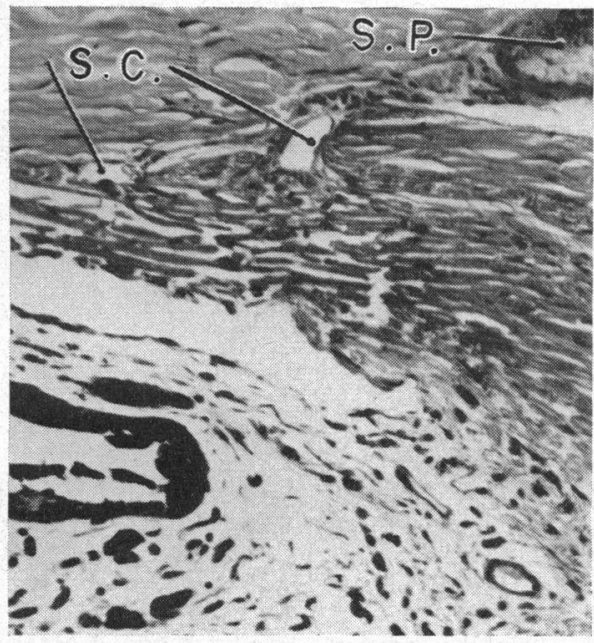

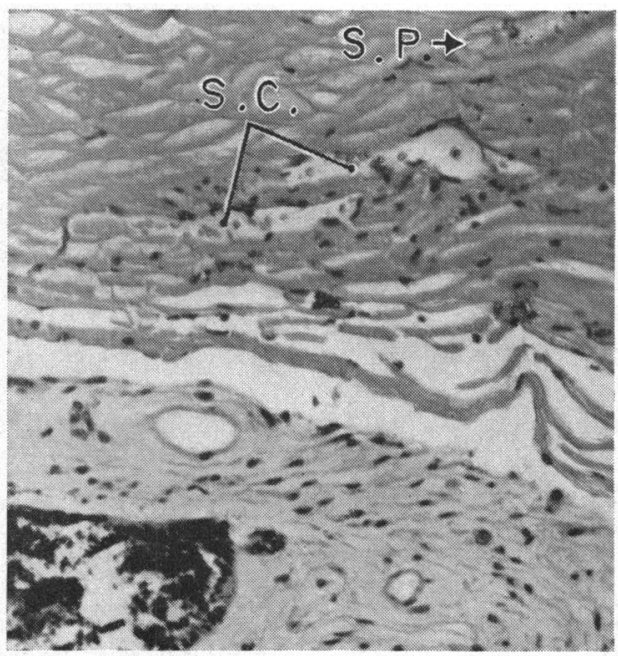

(b)

Fig. $2(a, b)$.- Paraffin sections, showing open filtration angles $(a)$ in Case 1 and $(b)$ in Case 2, with red blood cells in Schlemm's canal (s.c.) and scleral plexus (s.p.). Haematoxylin and eosin $\times 300$.

\section{Examination of Filtration Angles by Light and Electron Microscopy}

\section{[1] Trabecular Meshwork}

Uveal Meshwork.-Flat preparations obtained from the first eye showed under low magnification the typical lace-like pattern of openings (Fig. 3).

FIG. 3.-Flat preparation of uveal meshwork in Case 1, showing characteristic openings. Polychrome methylene blue $\times 300$.

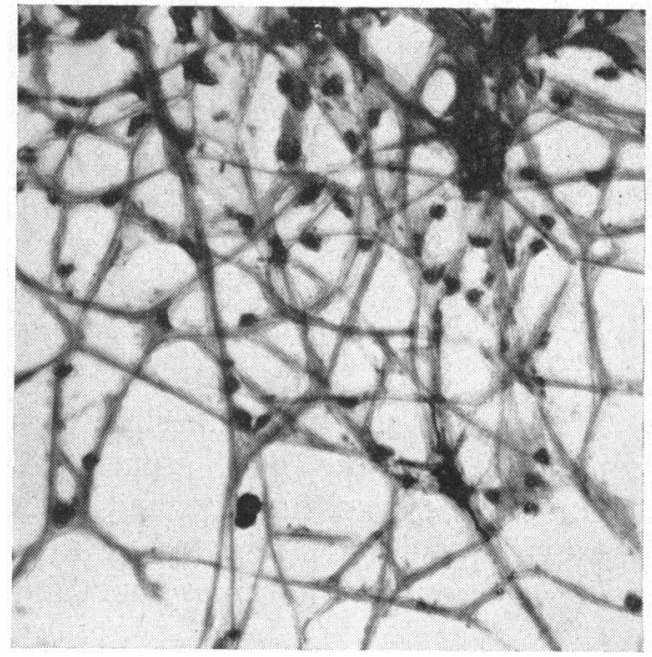

Under higher magnification very few normal fibres were found. The pathology consisted of an intensification of the staining of the fibre bundles forming the central core of collagen, some of which were found to terminate abruptly 
(Fig. 4a) while others showed varying signs of looping and twisting. In an early stage of development the loops were small (Fig. 4b), whereas in a more advanced stage the remains of core fibre bundles could be seen coiled inside large nodules (Fig. $4 c, d$ ).

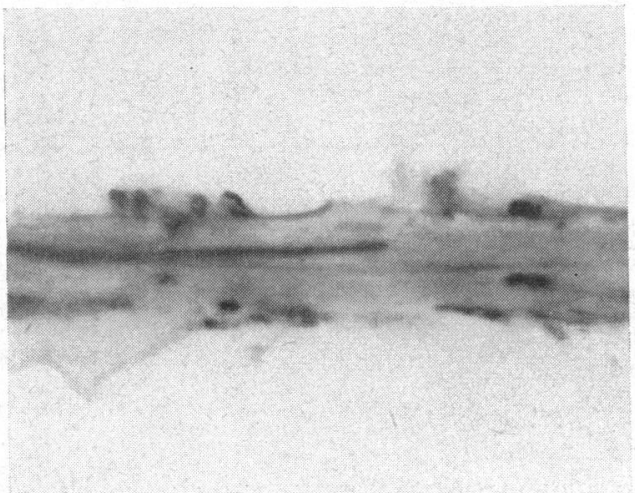

(a)

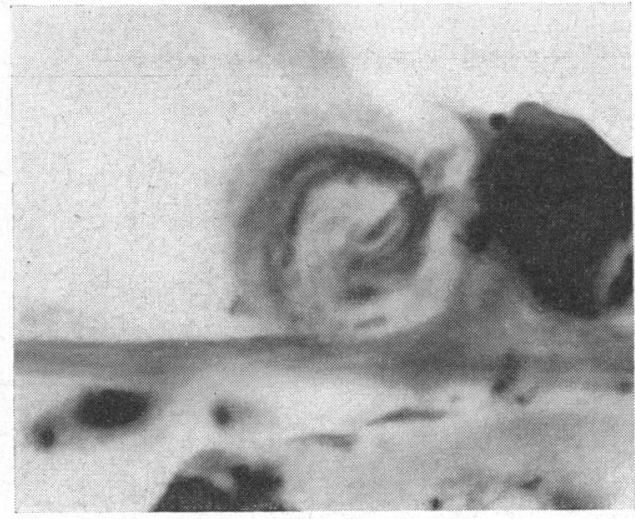

(c)

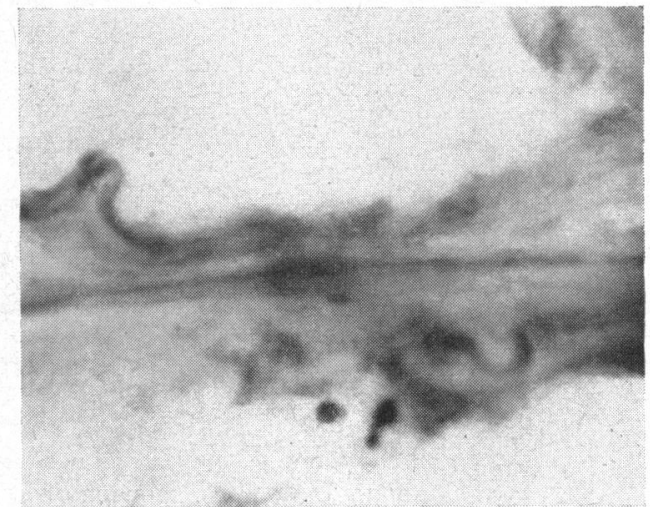

(b)

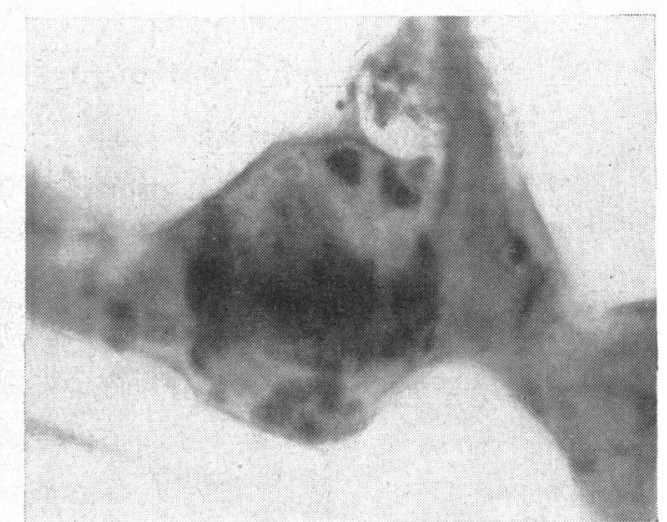

(d)

Fig. $4(a-d)$.-Flat preparations of uveal meshwork in Case 1, showing nodules at several stages of development and increased staining, fragmentation, looping and coiling of the core fibre bundles. Polychrome methylene blue. $\times 1,500$.

The clear zone surrounding the uveal fibres was irregular in width and contained numerous dark granules. The expansions of the clear zone assumed the typical fusiform and nodular shapes described previously and the dark granules appeared to be due to increased staining and coiling of the spiral fibre bundles normally found in this zone (Speakman, 1962). The nodules were found singly or in clusters, and when a group was packed closely together on the surface of a fibre the normal architecture was grossly distorted. No electron microscopic sections of uveal meshwork were obtained from the first eye and in the second eye the uveal fibres had almost completely disappeared except for occasional remnants consisting of a column 
of strongly eosinophilic nodules which projected into the anterior chamber from the root of the iris. No definite abnormalities were seen in the uveal meshwork in meridional paraffin sections (Fig. 5a), although in tangential sections typical nodules were present (Fig. $5 b$ ).

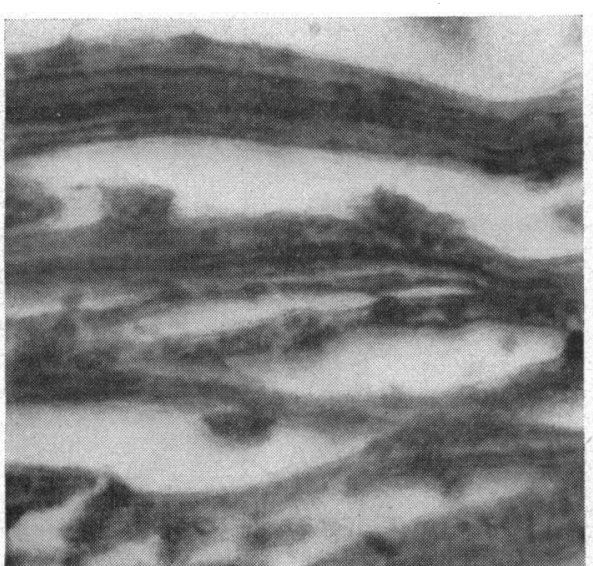

FIG. 5 (a).-Meridional paraffin section in Case 1, showing normal appearing uveal and corneo-scleral fibres.

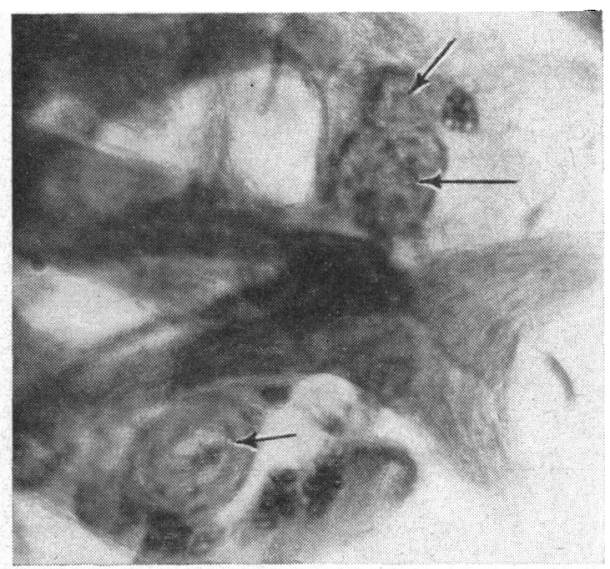

FIG. $5(b)$.- Tangential paraffin section in Case 1 , showing typical nodules (arrow) on uveal fibres. Haematoxylin and eosin. $\times 1,200$

Corneo-scleral Meshwork.-Flat preparations revealed the usual pattern of overlying lamellae perforated by oval and spiral openings. There was no evidence of cell proliferation, although some of the nuclei in the second eye were spindle-shaped or irregular and there was an increased deposition of pigment. In a few areas the spaces between lamellae resembled large vacuoles (Fig. 6), but this was not a striking feature of the meshwork and similar areas have been found in normal eyes.

FiG. 6.-Flat preparation of corneo-scleral meshwork in Case 1, showing spaces resembling vacuoles between adjacent lamellae. Polychrome methylene blue. $\times 1,500$.

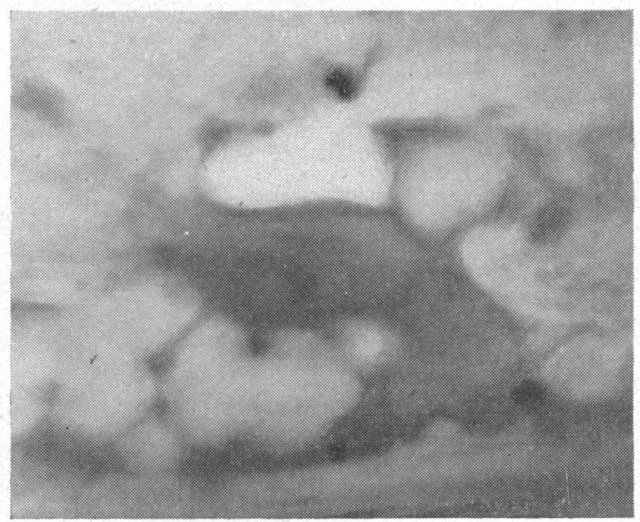

Under higher magnification a variable thickening of the clear zone was present throughout the meshwork of each eye. In the inner layers large and 
small nodules projected into the drainage channels between lamellae and many of these contained dark granules or coiled filaments (Fig. 7a,b).

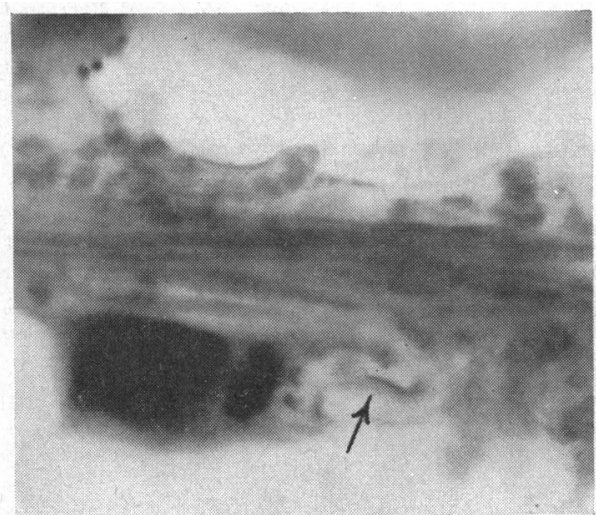

(a)

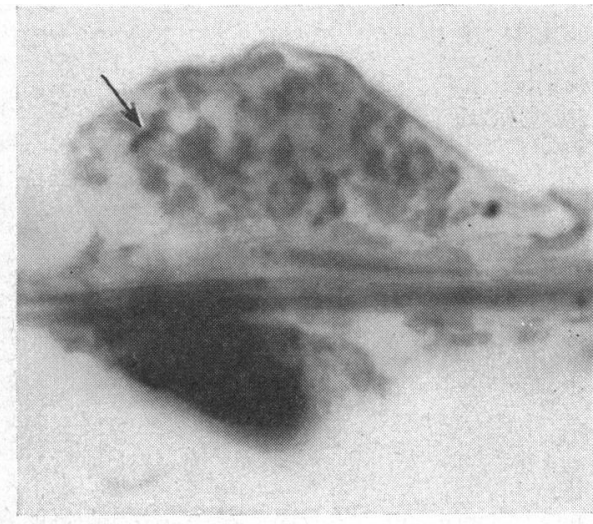

(b)

FIG. $7(a, b)$.-Flat preparations of corneo-scleral meshwork in Case 1, showing diffuse and nodular thickening of the clear zone. Many of the "granules" in the clear zone may be resolved into coiled hyperchromatic fibre bundles (arrow). Polychrome methylene blue. $\times 1,500$.

In the second eye nodules were less granular and although most retained a laminated structure others appeared amorphous. Some of the nodules in the second eye measured up to $15 \mu$ in diameter and these closely resembled Hassall-Henle bodies (Fig. 8).

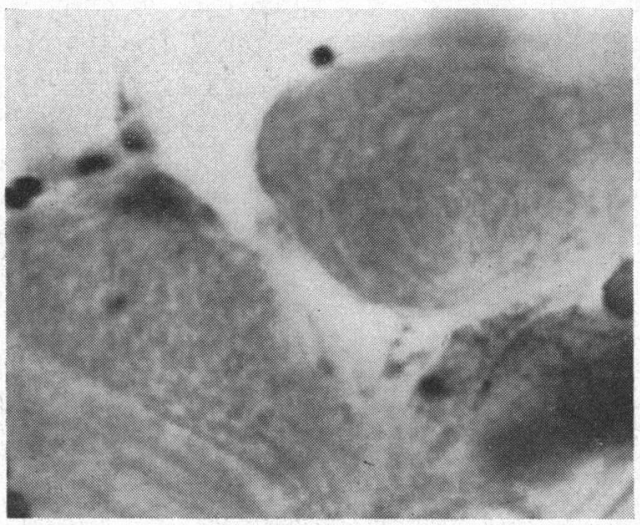

Fig. 8.-Flat preparation in Case 2, showing two large laminated nodules. Polychrome methylene blue. $\times 1,500$.

The nodules in the outer layers of the meshwork were smaller and appeared as a series of fine granules on the surface of the lamellae or as a thickening of the individual fibre bundles near Schlemm's canal. In meridional paraffin sections (Fig. 5a) it was difficult to recognize any abnormality in the meshwork whereas tangential sections showed thickening of the clear zone and nodule formation. 
Electron microscopic examination showed that the thickening of the corneo-scleral lamellae was the result of a massive proliferation of the longspacing collagen in the clear zone (Figs 9 and 10, overleaf). In normotensive eyes this material was found in isolated clumps or bundles intermingled with unit fibres of collagen and amorphous ground substance, and showed a major periodicity of 800 to $1200 \AA$ units with small nodules or fine connecting fibrils interposed between the dark bands (Leeson and Speakman, 1961). Although the unit fibres are stained (i.e. show an increase in electron density) by phosphotungstic acid, the long-spacing material is not. In both the glaucomatous eyes examined, this material to a great extent replaced or displaced the unit fibres of collagen normally present in the clear zone and in many sections appeared to be replacing or breaking up the core fibre bundles (Fig. 9a). In some cases thin sections were obtained through small nodular expansions and these were seen to consist almost entirely of bundles of long-spacing collagen (Fig. 9b). In sections from both eyes there were areas in the meshwork where the beams were of increased diameter with a corresponding reduction in the size of the trabecular spaces, but in the second eye this change was so gross that many of the trabecular spaces were completely obliterated and only a thin layer of cytoplasm separated adjacent lamellae (Fig. 10a).

In the first eye the long-spacing collagen showed identical appearances and staining reaction to those found in normotensive eyes and was merely increased in amount(Fig. $9 b, c)$. In the second eye there were areas where this material had undergone degenerative change. The typical dark cross-banding was not evident and the material occupying the clear zone appeared granular and disorganized (Fig. 10b, c). As with long-spacing collagen elsewhere, this granular material was unstained by phosphotungstic acid.

In both eyes the endothelial cells appeared more active than those observed in normotensive eyes. Mitochondria were numerous, the Golgi zone was more extensive and, particularly in the second eye, pigment granules were increased in number. In some sections the endothelial cytoplasm contained fine fibrillar material.

Endothelial Meshwork.-This lined the inner wall of Schlemm's canal and was easily identified in flat preparations by the characteristic spaces between the cells, the spindle-shaped nuclei, and the many red blood cells lying on the surface of the canal and in the underlying trabecular spaces. There was no evidence of cell proliferation, and the spaces in the endothelial lining were empty and showed the usual size variation and configuration (Fig. 11, overleaf, p. 332). One preparation showed many clearly-defined openings into the meshwork, and the collagen fibre bundles forming the lamellae in this preparation and others from the same region were thickened. In the second eye satisfactory flat preparations of the inner wall of the canal could not be obtained because of difficulty in identifying the lumen of the canal by gross dissection. 
(a)

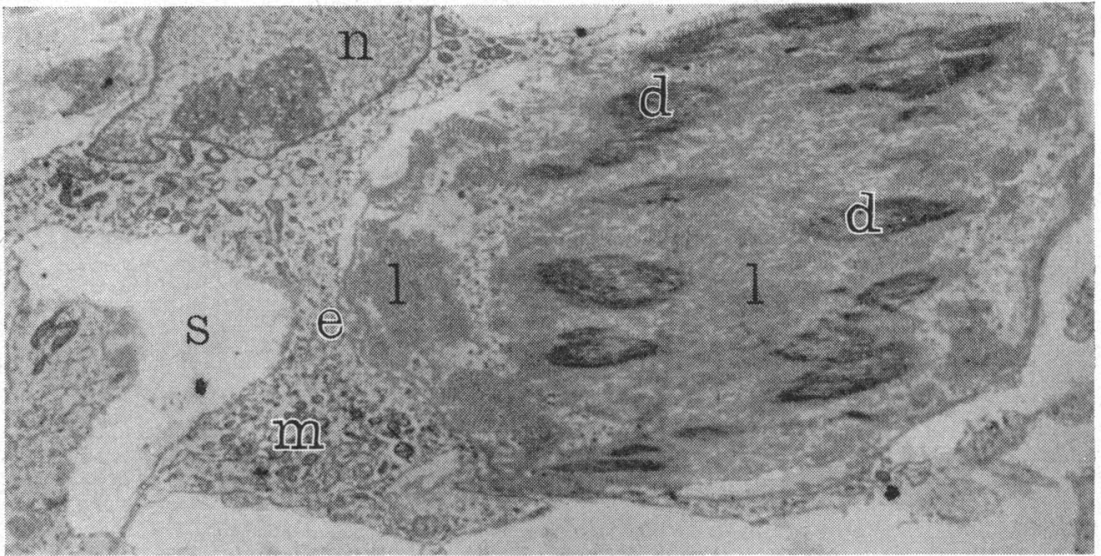

(b)

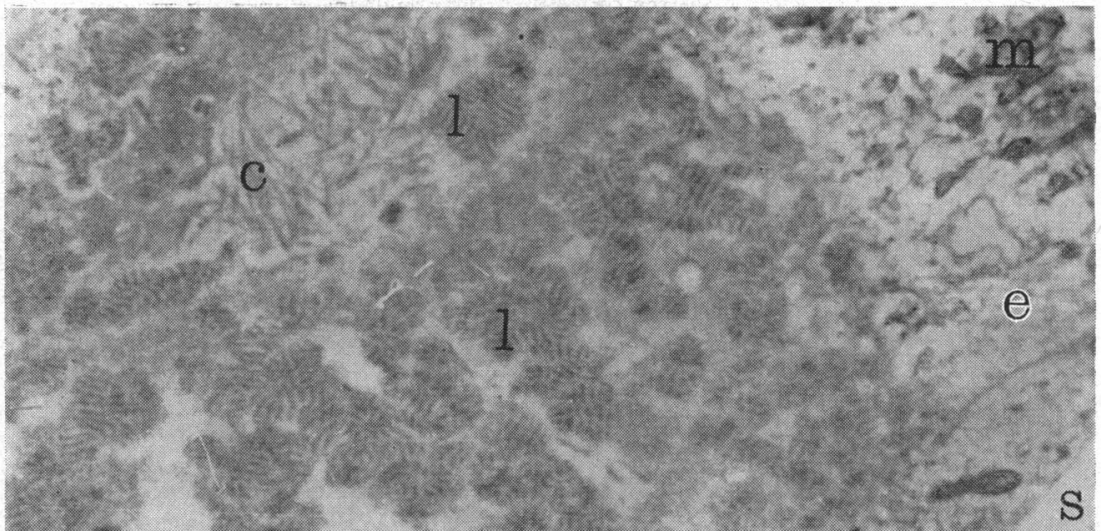

(c)

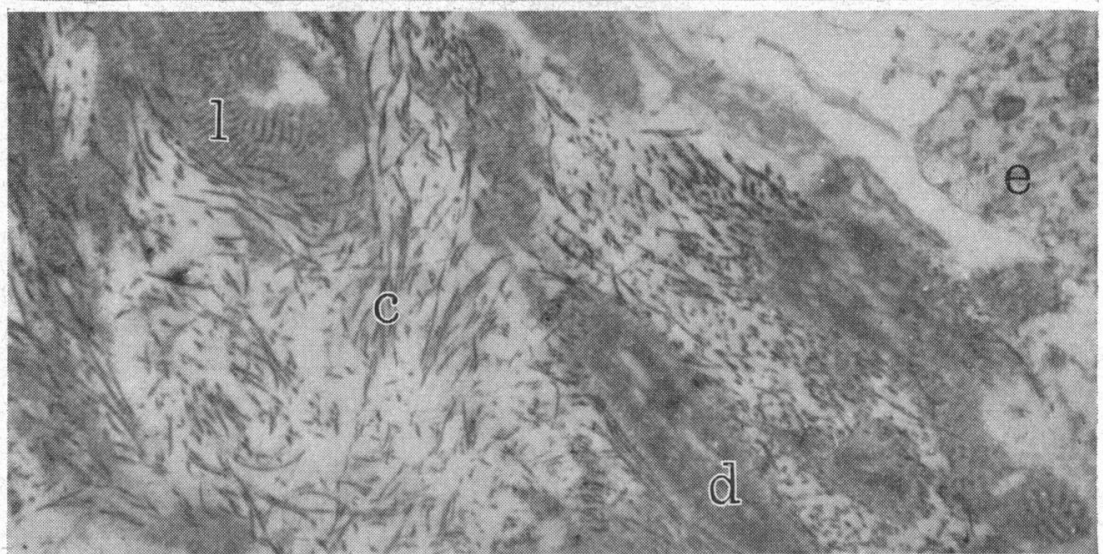

Fig. 9 (a).-Survey picture (Case 1), showing one beam of meshwork in cross-section covered by attenuated endothelial cytoplasm (e), with nucleus (n), and mitochondria (m). In the beam there are bundles of long-spacing collagen (1) both in the clear zone and in the core, surrounding masses of dense homogeneous material (d). $\times 3,466$.

Fig. 9 (b).-Part of one nodular swelling, showing numerous bundles of long-spacing collagen (1) interspersed with a few unit fibrils of collagen (c). $\times 7,626$.

FIG. $9(c)$. -Edge of a beam, covered by endothelium (e), with bundles of long-spacing collagen (l) unit fibrils (c), and dense material (d). Section stained with phosphotungstic acid. $\times 7,626$.

All sections of osmium-tetroxide-fixed, methacrylate embedded material unless otherwise stated.
a. abnormal, granular, long-spacing collagen. $\quad$ b. unit fibrils of collagen. 
Fig. 10 (a).- Survey picture (Case 2), showing compressed beams with virtually no trabecular spaces (s), the beams being separated by thin slips of endothelial cytoplasm (e). Some dense material (d) is present, but the clear zone is filled with granular, abnormal long-spacing collagen (a). $\times 3,766$.

FIG. $10(b)$.-Edge of such a beam at higher magnification. $\quad \times 7,626$.

FIG. $10(c)$.- Similar area at higher magnification, but of permanganate-fixed, Epon-embedded material. Note "wavy" nature of dark bands of long-spacing collagen (1). $\times 47,000$.

e. endothelial cytoplasm.

n. nucleus.
1. long-spacing ("curly") collagen.

p. melanin granule. m. mitochondria.

s. trabecular space.

(a)

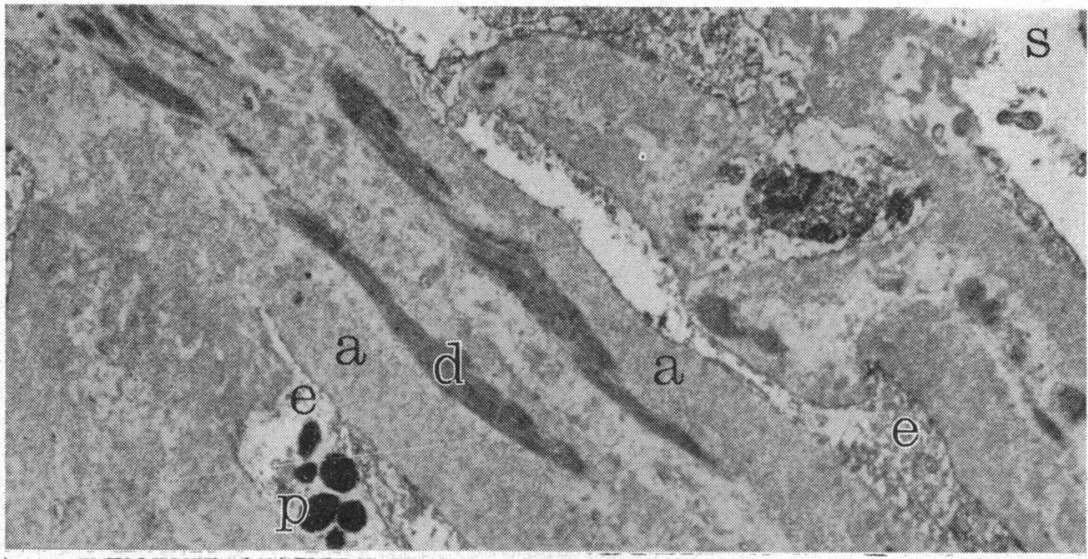

(b)

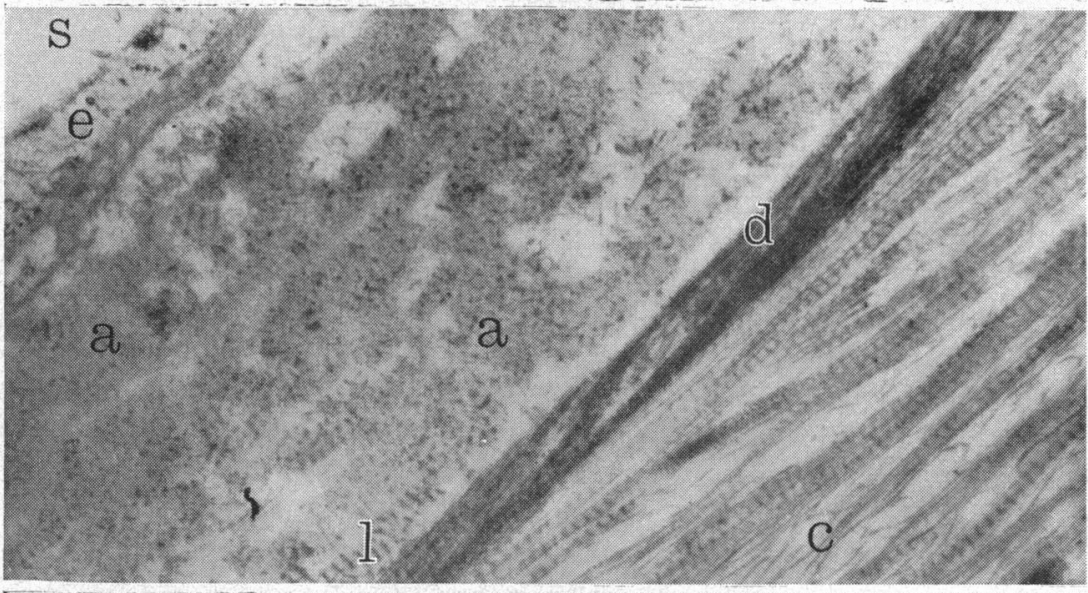

(c)

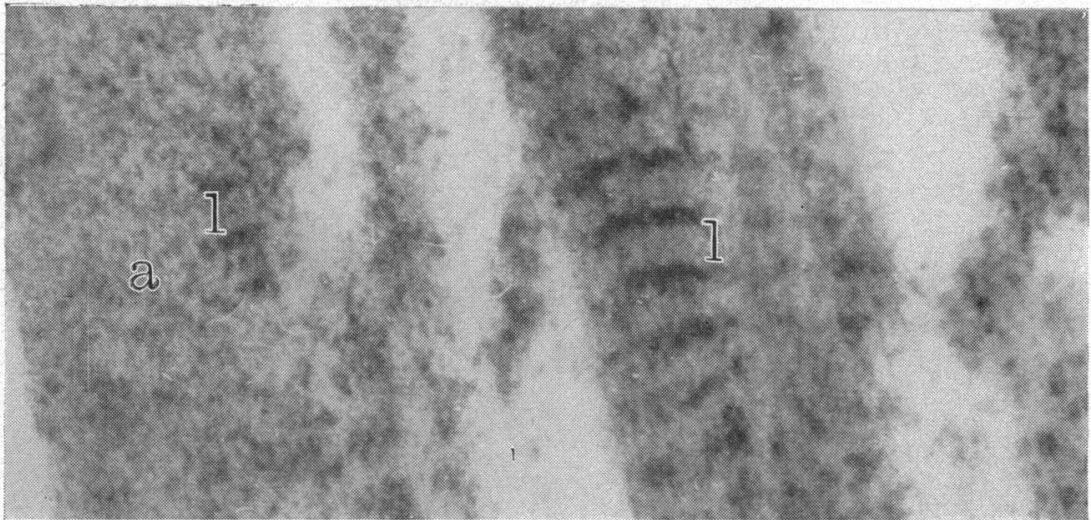




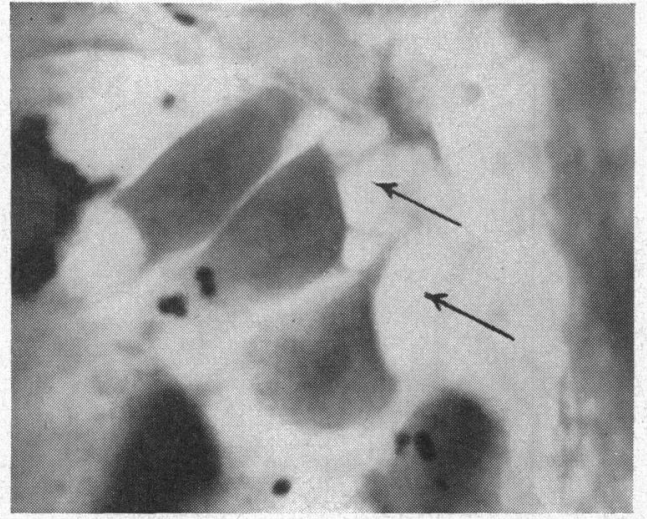

FIG. 11.-Flat preparation (Case 1), showing typical empty spaces (arrows) in the endothelial meshwork which forms the inner wall of Schlemm's canal. Polychrome methylene blue. $\times 1,500$.

\section{(2) Schlemm's Canal and Scleral Plexus}

In both eyes Schlemm's canal was open and contained red blood cells (Fig. $2 a, b)$. In the first eye many of the red cells had penetrated into the outer layers of the meshwork. The scleral plexus was not injected artificially but the decompression of the globe at the time of enucleation produced a marked influx of blood into the anterior segments which distended the scleral and episcleral vessels in both eyes. There was no evidence to indicate obliteration of these vessels. Pigment granules (presumably uveal in origin) were found among the red blood cells and within neutrophils lining the walls of the plexus. Clumps of pigment were also present in the perivascular connective tissue (Fig. 12).

Fig. 12.--Paraffin section (Case 1), through patent blood-filled vessels in the scleral plexus. Clumps of pigment (arrow) lie in the perivascular connective tissue. Haematoxylin and eosin. $\times 750$.

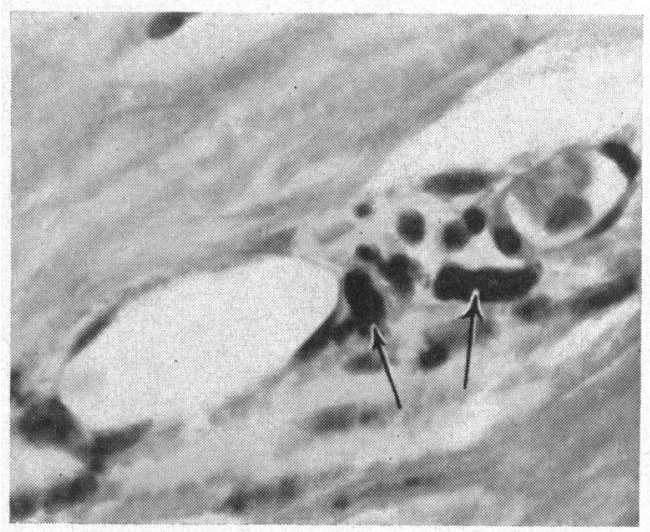

\section{Examination of the Posterior Segments}

Each optic nerve-head showed cupping and nasal displacement of the central retinal vessels which were open and contained blood. The peripheral 
retinal blood vessels were also patent and no haemorrhages or exudates were seen in the retina in the first eye. In the second eye a large amount of exudate filled the outer plexiform layer in the macular region and in the peripheral retina several small retinal haemorrhages were present. In the first eye ganglion cells were found in the nasal half of the retina but in the second eye the ganglion cells had disappeared. The rods and cones, pigment epithelium and choroid appeared healthy. Both lenses were examined in paraffin sections and no evidence of capsule exfoliation was found.

\section{Discussion}

Two eyes from patients with chronic simple glaucoma have shown a similar nodular thickening of the trabecular meshwork which was due to a massive proliferation of the long-spacing collagen in the clear zone. In the younger patient with more advanced glaucoma, the newly-formed material had undergone a granular degenerative change and the trabecular spaces were partially obliterated. It has already been established by light microscopy that a nodular dystrophy consisting of expansions of the clear zone of trabecular fibres may involve limited areas of the meshwork in eyes with normal intra-ocular pressure in older individuals (Speakman, 1961, 1962). A survey by electron microscopy has shown that there is a gradual increase with age in the size and number of the long-spacing collagen bundles in the clear zone which lies between the covering layer of endothelium and the central collagen core (Leeson and Speakman, 1961). The degree and extent of these proliferative and degenerative changes in the glaucomatous material have not been seen previously in eyes with normal intra-ocular pressure. This evidence suggests that the raised intra-ocular pressure in the glaucomatous eyes was the result of the pathological changes found in the trabecular meshwork. The absence of any demonstrable obstruction in the canal of Schlemm or scleral plexus and the free flow of blood through these channels support this view.

The entire sequence of events in the meshwork which leads to ocular hypertension in glaucoma cannot be determined with certainty because the number of eyes available for study has been small and knowledge of the factors controlling collagen synthesis is incomplete. Nevertheless, as a result of an environmental stimulus or possibly of a change within the trabecular fibres themselves, the endothelial cells covering the trabecular fibres lay down more long-spacing collagen in the inner layers of the meshwork. The fragmentation, twisting, and curling of the older collagen fibre bundles in the core and clear zone may be the first change to take place, and the increased fibre synthesis may occur in response to a weakening or degeneration of the preexisting collagen. The proportion of the meshwork involved increases and the end result is an almost complete replacement and expansion of the clear zone and a partial invasion of the core region by long-spacing collagen. The normal architecture of the trabecular fibres is distorted and the calibre of the 
drainage channels is reduced. Eventual destruction of the trabecular fibres takes place, as indicated by the absence of the uveal meshwork in the second eye. The obliteration of the trabecular spaces, the distortion of endothelial cell nuclei, and the granular degenerative change of the long-spacing collagen, may be secondary to raised intra-ocular pressure, and may represent the final stages of the dystrophy. The increased accumulation of pigment in the meshwork can be attributed to a more sluggish flow of aqueous and this no doubt contributes to the obstruction when excessive. The observation of pigment granules in phagocytic cells lining the scleral plexus and in clumps in the extravascular connective tissue, suggests the possibility that in the trabecular spaces and scleral plexus there is a constant removal of particulate matter by phagocytic cells passing through the meshwork with the aqueous. The disposal of uveal pigment suggests that debris in the meshwork may also be carried into the blood stream or deposited in the extravascular connective tissues.

The nodular proliferations described in this paper were recognized by Salzmann (1912) in flat teased preparations. He noted spindle-shaped thickenings of the trabecular fibres but did not attach any significance to this observation. Since then there has been no detailed study made of flat preparations and the proliferative changes in the meshwork appear to have been overlooked because in routine meridional paraffin sections the trabecular beams are cut at right-angles and the proliferative changes cannot be identified. The chances of sectioning a recognizable nodule by this technique are rare and the diffuse thickenings and fusiform expansion of the fibres cannot be differentiated from irregularities in the contour of lamellae which occur normally. For this reason it seems doubtful whether the nodular proliferative changes have previously been recognized in those reports based on a study of routine paraffin sections. The proliferative changes are clearly evident in tangential sections which cut the trabecular beams lengthwise, and nodules, which Wolter (1960) found in flat frozen sections and attributed to shrinkage, appear to be similar to the pathology described in this paper. The thickening of the clear zone described by Unger and Rohen (1960) in trephine biopsies appears more uniform than the nodular thickening described here. These and other investigators have also described endothelial cell proliferation, which was not confirmed by this investigation, nor did our findings support the reports of Becker (1959) and Flocks (1959) who described cystic or foamy degeneration of the meshwork. The thickening of the uveal fibres in flat preparations reported by Ashton and Speakman (1959) in a case of glaucoma was similar and was thought at the time to be due either to post mortem shrinkage (fixation was delayed for several hours) or to swelling of the ground substance, whereas this study has shown that the thickening of the trabecular fibres is due to a true proliferation of extra-cellular fibres. The only electron microscopic report of a case of glaucoma is that of Garron (1959a) and his finding of an increase in curly collagen or long-spacing collagen in the trabecular meshwork 
is identical to our own observations. Clinical details of his case were not recorded, however, and the patient had either macular degeneration or a heàled choroiditis as well as chronic glaucoma.

Although this report records the examination of only two glaucomatous eyes, the accumulated evidence, based on the similarity of the findings in each eye, the occurrence of identical but less extensive pathology in normotensive eyes, the study of normal eyes at different ages, and the findings reported in the literature, indicates that raised intra-ocular pressure in chronic glaucoma is the result of a progressive nodular dystrophy in the trabecular meshwork. Whether or not this is the only cause of primary chronic glaucoma can only be resolved by the examination of additional eyes. One would also expect the coexistence of a nodular dystrophy in the meshwork to contribute to obstruction to aqueous outflow when secondary conditions predominate. This may explain why patients with the same degree of trabecular pigmentation or lens exfoliation do not all have glaucoma, as the nodular proliferations cannot be detected clinically, if present on the fibres of the meshwork.

\section{Conclusion}

The pathological findings in two eyes from patients with chronic simple glaucoma support the hypothesis that the increased intra-ocular pressure found clinically was the result of a progressive dystrophy of the trabecular meshwork, consisting primarily of a diffuse nodular proliferation of extràcellular long-spacing collagen. The proliferative changes were associated with fragmentation and coiling of the fibre bundles, partial destruction of the trabecular fibres, and obliteration of the trabecular spaces.

\section{REFERENCES}

AsHton, N. (1960). Trans. ophthal. Soc. U. $\dot{K} .$, 80, 397. and SPeAKMan, J. S. (1959). Proc. roy. Soc. Med., 52, 69 and 72.

BECKER, B. (1959). In "Glaucoma: Trans. IV Conf.", p. 223. Josiah Macy, Jr., Foundation, New York.

Duke-EldER, S. (1955). In “Glaucoma: A Symposium”, ed. S. Duke-Elder, p. 147. Blackwell, Oxford.

Flocks, M. (1959). Amer. J. Ophthal., 47, 519.

GARRON, L. K. (1959a). In “Glaucoma: Trans. IV Conf.”, p. 231. (1959b). Ibid., p. 11.

GoldmanN, H. (1949). Trans. ophthal. Soc. U.K., 69, 455.

Grant, W. M. (1958). A.M.A. Arch. Ophthal., 60, 523.

HolmberG, A. S. (1959). In "Glaucoma: Trans. IV Conf.", p. 179.

Kornsweig, A. L., Feldstein, M., Schneider, J. (1958). Amer. J. Ophthal., 46, 311.

LeESON, T. S., and SPEAKman, J. S. (1961). Acta anat. (Basel), in the press.

PERT (1962). Nature (Lond.), 193, 694.

Perkins, E. S. (1955). Brit. J. Ophthal., 39, 215.

SalzmanN, M. (1912). "The Anatomy and Histology of the Human Eyeball", trans. E. V. L. Brown, p. 48. University of Chicago Press.

SPEAKMAN, J. S. (1959). Brit. J. Ophthal., 43, 129.

(1960). Ibid., 44, 513.

(1961). Canad. med. Ass. J., 84, 1066.

(1962). Brit.J. Ophthal., 46, 31.

Teng, C. C., Parton, R. T., and Katzin, H. M. (1955). Amer. J. Ophthal., 40, 619.

THEOBALD, G. DVORAK, and KIRK, H. Q. (1956). Ibid., 41, 11.

UNGER, H. H., and ROHEN, J. (1960). Ibid., 50, 37.

Wolter, J. R. (1960). Ibid., 49 (No. 5, pt 2, pt 3), 1089. 